\title{
Scale Determination Using the Static Potential with Two Dynamical Quark Flavors
}

\author{
Sonali Tamhankar* and Steven Gottlieb \\ Indiana University, Bloomington, IN 47405, USA
}

We study the static potential using gauge configurations that include the effects of two flavors of dynamical Kogut-Susskind quarks. The configurations, generated by the MILC collaboration, and used to study the spectrum and heavy-light decay constants, cover a range $5.3 \leq 6 / g^{2} \leq 5.6$. There are at least four quark masses for each coupling studied. Determination of $r_{0}$ from the potential can be used to set a scale. This alternative scale is useful to study systematic errors on the spectrum and decay constants.

\section{INTRODUCTION}

To get an idea of systematic errors introduced in determining the physical scale in lattice QCD simulations using $m_{\rho}$, it is important to use an alternative quantity to set the physical scale. One quantity, $r_{0}$, proposed by Sommer [1] has recently been studied in several papers 2 - 4 . Here, we examine the static potential for a range of couplings $5.3 \leq 6 / g^{2} \leq 5.6$ with two flavors of dynamical staggered quarks. We compute the Sommer scale for each dynamical quark mass and extrapolate to the chiral limit at each coupling. Finally, using the scale determined by $r_{0}$, we take the continuum limit and replot the Edinburgh curve, finding very little difference as compared with setting the scale from $m_{\rho}$.

The configurations were generated by the MILC collaboration [5] using the staggered quark action with two dynamical flavors and the Wilson gauge action. They were stored every 10 units of molecular dynamics time. These configurations have been used elsewhere for light hadron spectrum calculations [6]. For each coupling there are at least four quark masses. (For details, see Ref. [7].)

\section{STATIC POTENTIAL}

To calculate the static potential, Wilson loops $W(R, T)$ were measured for 5 and 10 smearing steps using the APE smearing method as ex-

\footnotetext{
* presented by S. Tamhankar
}

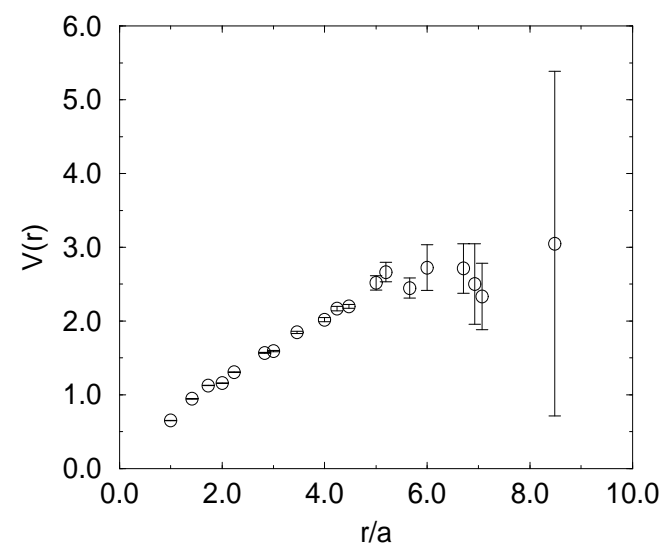

Figure 1. Hint of string breaking: flattening of the last few points. $\beta=5.3, a m_{q}=0.075$.

plained in [8]. The effective potentials were then calculated using

$V_{T}(R)=\ln \left[\frac{W(R, T)}{W(R, T+1)}\right]$

After blocking the results into 30 time unit blocks, errors are obtained using single elimination jackknife. $V(R)$ is expected to flatten out at large $R$ for dynamical quarks due to string breaking. At our strongest coupling, 5.3, and smallest quark masses we have searched for evidence of string breaking. For the two lightest masses, we did not find a signal at large enough values of $R$, but we do find a hint of string breaking for $a m_{q}=0.075$. In Fig. 1, the potential seems to flatten for $R$ greater than five to seven lattice spacings. This 

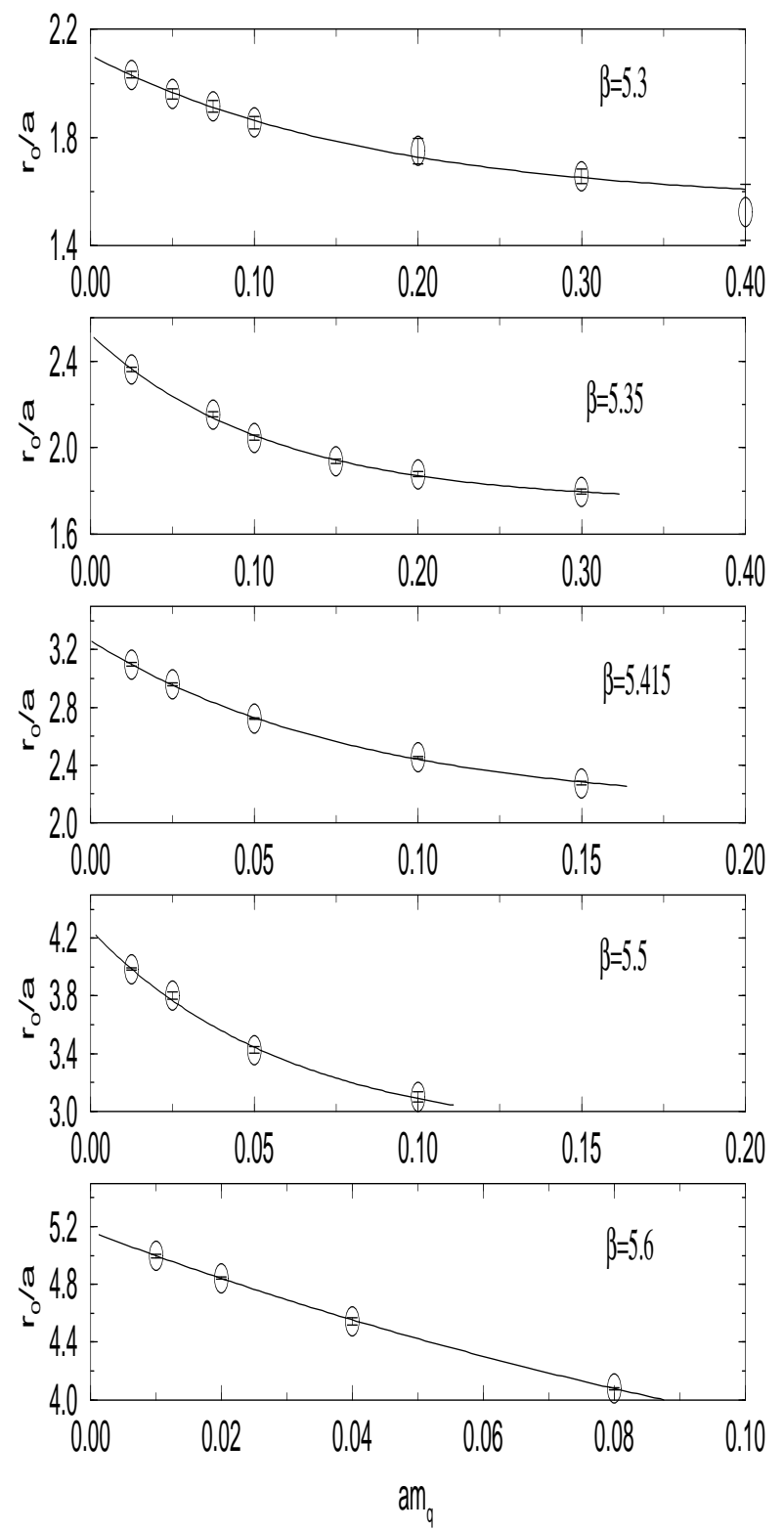

Figure 2. The graphs show $r_{0} / a$ vs. $a m_{q}$ for different $\beta$ values, along with an exponential fit defined in Eq. (4).

corresponds to a distance of $1.3-1.8 \mathrm{fm}$. Ref. [9] reports finding signs of string breaking at $0.8-1.1$ $\mathrm{fm}$, however their $m_{\pi} / m_{\rho}$ is lower. This is consistent: the string is expected to break at smaller distances for lighter quarks.

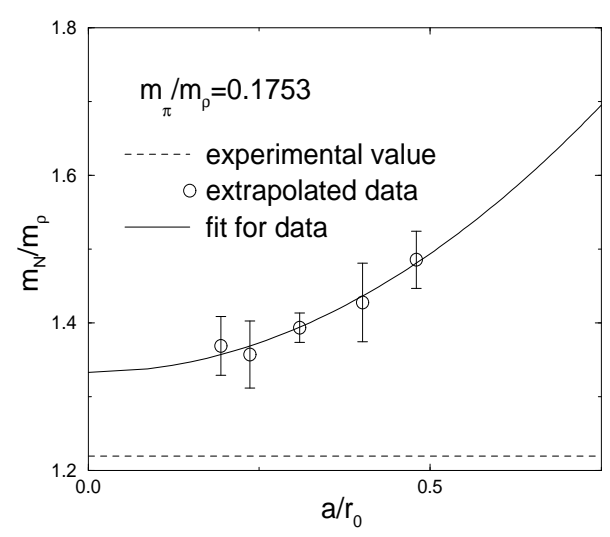

Figure 3. Continuum extrapolation for $m_{\pi} / m_{\rho}=$ 0.1753. Continuum extrapolation was made at various $m_{\pi} / m_{\rho}$ values to plot the Edinburgh curve shown in Fig. 4

\section{FIT PARAMETERS}

We used the standard ansatz for the potential,

$V(R)=V_{0}+\sigma R-e / R-f\left(G_{L}-1 / R\right)$.

This is a simple combination of a Coulomb term at short distances and a linear increase at large distances. The last term accounts for lattice artifacts. For more details on this ansatz and its limitations see Refs. [2] and 10].

Using the fit parameters $\sigma$ and $e$, the Sommer scale $r_{0}$ is calculated in the usual way,

$r_{0}=\sqrt{\frac{c-e}{\sigma}}$

with $\mathrm{c}=1.65$. This corresponds to a physical length of $r_{0} \sim 0.5 \mathrm{fm}$.

We fit the potential to the ansatz (2) for different ranges of $R$ and different values of $T$. The "good" fits yielded consistent values, except for $\beta$ $=5.35, a m_{q}=0.05$. We have excluded this point from our fits for the chiral extrapolation. The criteria for best fits were the confidence of the fit and the degrees of freedom. Additionally, the range of $R$ had to include the value of $r_{0}$, which was an important constraint for the coarse lattices. In Fig. 2, we show the best estimates of $r_{0}$ along with the exponential fit explained below. 


\section{SCALE DETERMINATION}

For dynamical quarks, a different value of $r_{0}$ and $\sigma$ is obtained for each quark mass. The consideration that the physical scale should approach the quenched result as the quark mass tends to infinity and the graphs of $r_{0}$ vs $a m_{q}$ suggest an exponential fit

$r_{0}=A+B\left(e^{-\operatorname{Cam}_{q}}-1\right)$

with three parameters $A, B$ and $C$.

We obtained good fits to this form for all $\beta$ values except 5.415 , where we excluded the heaviest two points from the fit to get an acceptable confidence level. These fits were used for the chiral extrapolation to determine $r_{0}$ for each $m_{q}$.

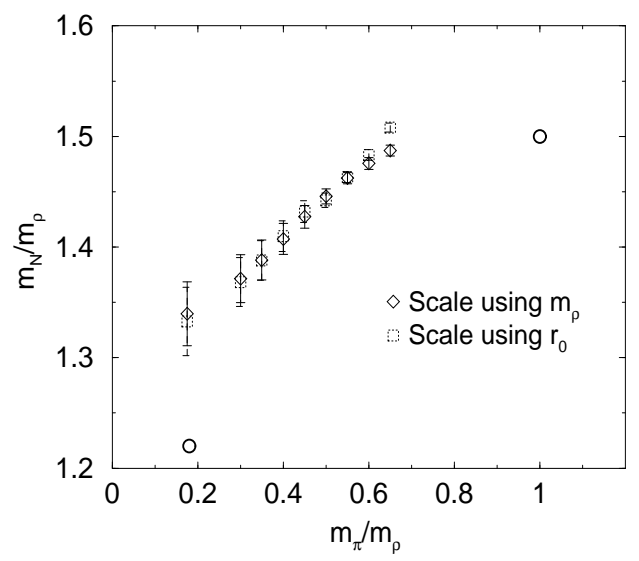

Figure 4. Continuum Edinburgh curves for dynamical fermions using the scale as determined by $m_{\rho}$ and $r_{0}$.

\section{EDINBURGH PLOT}

A most surprising result of Lattice '98 was that the Edinburgh plot for dynamical quarks was shifted up compared to the quenched simulations, leading to a larger deviation from the real world value in the continuum limit [6]. We were curious to know if the Edinburgh curve changed when setting the scale from $r_{0}$. Fig. 3 shows $m_{N} / m_{\rho}$ as a function of $a / r_{0}$ for the real world $m_{\pi} / m_{\rho}$ value.2 The Edinburgh curve is replotted using

${ }^{2}$ These fits were good up to $m_{\pi} / m_{\rho}=0.45$ but for higher ratios the $\beta=5.415$ point departed from the curve. There the scale determined here in Fig. 4.

\section{CONCLUSIONS}

We have calculated the heavy quark potential for a range of couplings for dynamical staggered quarks. Some flattening of the static potential for large $R$ at strong coupling is seen which hints at string breaking, but does not suffice to make a conclusive case. We determine $r_{0}$, extrapolate it to the chiral limit using an exponential form and examine the hadron mass data using the new scale. We do not find significant deviations from the previous continuum extrapolations using the $\rho$ mass to set the scale.

We would like to thank the rest of the MILC collaboration for the use of the lattices and U.M. Heller for some fitting routines. We are grateful to the US Department of Energy which supported this work under grant FG 02-91ER40661. The computations were performed on the T3E at PSC, Origin-2000s at NCSA and an Origin-2000, a Paragon and the CANDYCANE Linux cluster at Indiana University.

\section{REFERENCES}

1. R. Sommer, hep-lat/9310022.

2. R.G. Edwards et al., hep-lat/9711003v2.

3. U.M. Heller et al., Phys. Lett. B335, (1994) 71.

4. C.R. Allton et al., hep-lat/9808016, and references therein.

5. C. Bernard et al., Nucl. Phys. B(Proc. Suppl.) 60A (1998) 297.

6. C. Bernard et al., hep-lat/9810035.

7. S. Tamhankar and S. Gottlieb, in preparation.

8. M. Albanese et al., Phys. Lett. 192B (1987) 163.

9. C. DeTar et al., these proceedings.

10. C. Michael, Phys. Lett. 283B (1992) 103.

seems to be a problem with that coupling for higher masses that we do not completely understand yet. Hence we have excluded that point from the fits for continuum extrapolation for $m_{\pi} / m_{\rho}>0.45$. This exclusion yielded better fits without much shift in the continuum value. 